\title{
QUALIDADE DE MUDAS DE NIM INDIANO PRODUZIDAS EM DIFERENTES RECIPIENTES E SEU DESEMPENHO NO CAMPO
}

\author{
Adalberto Brito de Novaes ${ }^{1}$, Helane França Silva², Glauce Taís de Oliveira Sousa ${ }^{3}$, \\ Gileno Brito de Azevedo ${ }^{4}$ \\ Eng. Agrônomo, Dr., UESB, Vitória da Conquista, BA, Brasil - adalberto.brito@ globo.com \\ ${ }^{2}$ Eng $^{\mathrm{a}}$ Florestal, Mestranda em Engenharia Florestal, UFLA, Lavras, MG, Brasil - helane.engflo@gmail.com \\ ${ }^{3}$ Eng $^{\mathrm{a}}$ Florestal, Mestranda em Ciências Florestais, UnB, Brasília, DF, Brasil - gtosousa@ gmail.com \\ ${ }^{4}$ Eng. Florestal, Mestrando em Ciências Florestais, UnB, Brasília, DF, Brasil - gilenoba@ hotmail.com \\ Recebido para publicação: 13/12/2012 - Aceito para publicação: 06/11/2013
}

\begin{abstract}
Resumo
A presente pesquisa teve como objetivo avaliar a qualidade morfofisiológica de mudas de nim indiano (Azadirachta indica A. Juss) em diferentes recipientes, visando alcançar maior produtividade no campo. Estabeleceram-se quatro tratamentos: T1 - sacola plástica $\left(382 \mathrm{~cm}^{3}\right)$; T2 - sacola plástica $\left(165 \mathrm{~cm}^{3}\right)$; T3 - tubete $\left(288 \mathrm{~cm}^{3}\right)$; T4 - tubete $\left(55 \mathrm{~cm}^{3}\right) . \mathrm{O}$ experimento foi desenvolvido em três etapas distintas. A primeira consistiu na produção e avaliação morfológica das mudas, visando avaliar as variáveis altura da parte aérea $(\mathrm{H})$, diâmetro de colo $(\mathrm{D})$, relação $H / D$ e pesos de matéria fresca e seca das partes aérea, radicial e total. A segunda etapa objetivou a avaliação do potencial de regeneração de raízes (PRR) das mudas e a terceira fase foi desenvolvida no campo com o intuito de avaliar a sobrevivência das mudas após o plantio e o seu desempenho inicial, por meio da medição da altura e do diâmetro ao nível do solo. As mudas de nim indiano produzidas em sacolas plásticas $\left(382 \mathrm{~cm}^{3}\right)$ apresentaram valores superiores em todas as características morfológicas avaliadas e melhor desempenho no campo aos 15 meses após o plantio. O PRR se mostrou uma característica confiável quanto ao desempenho no campo de mudas produzidas em tubetes de $288 \mathrm{~cm}^{3}$.

Palavras-chave: Potencial de regeneração de raízes; sacolas plásticas; tubetes.
\end{abstract}

\begin{abstract}
Quality of Indian Lilac seedlings produced in different containers and their performance in field. The present research aims to evaluate the quality of Indian Lilac (Azadirachta indica A. Juss) seedlings in different containers in order to obtain higher productivity in the field. It was established four treatments: T1 - Plastic bags $\left(382 \mathrm{~cm}^{3}\right)$; T2 - Plastic bags $\left(165 \mathrm{~cm}^{3}\right)$; T3 - Tubes $\left(288 \mathrm{~cm}^{3}\right)$ and T4 Tubes $\left(55 \mathrm{~cm}^{3}\right)$. The experiment was developed in three different stages. The first one consisted on the production and morphological evaluation of the seedlings, seeking to evaluate the variables: stem height $(\mathrm{H})$, root-collar diameter $(\mathrm{D})$, ratio H/D and weights of fresh and dry matter of the stem, root and total. The second stage aimed at evaluation of root regeneration potential (RRP) of seedlings, and the third phase was developed in the field in order to evaluate seedlings survival after planting and their performance by measurement of the height and diameter at the soil level. The seedlings of Indian Lilac produced in plastic bags $\left(382 \mathrm{~cm}^{3}\right)$ presented superior values in all evaluated morphological parameters and improved performance in the field, 15 months after the planting. The RRP has proved a reliable parameter on the field performance of seedlings produced in tubes of 288 $\mathrm{cm}^{3}$.
\end{abstract}

Keywords: Root regeneration potential; plastic bags; tubes.

\section{INTRODUÇÃO}

O nim indiano (Azadirachta indica A. Juss) também conhecido por margosa ou neen, pertence à família Meliaceae. Tem como região de origem o sudeste da Ásia, mais precisamente Índia, Myanmar, Ceilão, Filipinas, Indonésia e Malásia (MARTINEZ et al., 2002; LORENZI et al., 2003). Encontra-se em vários países da África, ilhas asiáticas, Austrália e América do Norte, Central e do Sul, devido à sua grande rusticidade e adaptação a regiões áridas (MARTINEZ et al., 2002). Ainda segundo esses autores, 
no Brasil, o nim indiano foi introduzido para estudo como planta inseticida pelo Instituto Agronômico do Paraná (IAPAR).

Nos últimos anos, o nim indiano tem chamado muita atenção em função dos seus múltiplos usos, sendo seus produtos usados de forma mais intensa na agricultura, pecuária, medicina e cosméticos (BITTENCOURT et al., 2009). Trata-se de uma árvore que já apresenta boa aceitação nas regiões Norte, Sudeste, Centro-Oeste e agora no Nordeste brasileiro. Os plantios dessa planta têm possibilitado grande retorno financeiro aos produtores, devido a sua ampla utilização, como folhas desidratadas, madeira e, principalmente, extratos pesticidas oleosos (MOURÃO et al., 2004).

A azadirachtina, principal composto ativo do nim indiano, tem sido utilizada principalmente como inseticida. Na medida em que, ainda hoje, se usam muitos produtos químicos para o controle de pragas agrícolas sem orientação técnica, provocando sérios impactos ambientais, o óleo retirado das sementes dessa planta surge como uma alternativa em busca do controle de um grande número de pragas e doenças. Além dessas qualidades, sua madeira tem sido utilizada com muita propriedade na construção civil. Por apresentar grande durabilidade e resistência, é usada também na fabricação de postes para cerca, móveis, confecção de ferramentas e implementos agrícolas, além de ser excelente fonte de lenha e combustível, gerando carvão de alto poder calorífico (SOARES et al., s.d.).

O nim indiano é uma árvore que, normalmente, apresenta fuste reto e diâmetro entre 25 e $30 \mathrm{~cm}$ aos oito anos de idade. Cresce bem em regiões com precipitação pluviométrica anual entre 400 e $800 \mathrm{~mm}$. Todavia, já foi inserido com sucesso em áreas onde a precipitação está em torno de $250 \mathrm{~mm}$ anuais (MOURÃO et al., 2004). De acordo com Soares et al. (s.d.), a espécie se desenvolve bem em temperaturas acima de $20^{\circ} \mathrm{C}$, com altitudes superiores a $700 \mathrm{~m}$. Floresce, até mesmo, em solos pobres em nutrientes, porém não suporta locais encharcados e salinos, requerendo $\mathrm{pH}$ do solo de 6,2 a 7,0 para o seu bom desenvolvimento.

A espécie pode ser propagada via sementes, estaquia e cultura de tecidos (OLIVEIRA et al., 2005). Conforme Mourão et al. (2004), as mudas de nim indiano podem ser produzidas em sacos plásticos com uma boa irrigação, devendo as sementes ser semeadas a dois centímetros de profundidade, direto nos recipientes ou em sementeiras.

Para Neves et al. (2003), realizar a pré-germinação das sementes de nim indiano resulta em maior porcentagem de germinação. Nesse sentido, utilizam-se canteiros contendo areia, matéria orgânica e camadas finas de estopa úmida, sendo o período de pré-germinação em torno de uma semana, com as sementes apresentando radículas de aproximadamente dois milímetros, podendo, a partir desse momento, ser semeadas. Ainda de acordo esses autores, a semeadura direta em recipientes tem sido o método mais empregado para produção de mudas dessa espécie, principalmente pela eliminação da operação de confecção de canteiro para semeadura, redução do prazo para produção da muda, diminuição de perdas por doenças fúngicas, devido à menor concentração por unidade de área, e, finalmente, produção de mudas com menor custo unitário.

Segundo Vitorino et al. (1996), com a busca de maiores produtividades em áreas de plantios florestais, a qualidade das mudas tem sido intensamente pesquisada, no sentido de se estabelecerem dados que indiquem os melhores substratos, adubações e recipientes que proporcionem a qualidade de mudas desejada.

Conforme Goor (1964), o plantio de mudas em recipientes possibilita a formação de povoamentos florestais sob condições climáticas e edáficas adversas, assegurando maior desenvolvimento inicial das plantas. Para Carneiro (1995), as principais funções de um recipiente são: conter substrato que permita o crescimento e a nutrição das mudas, promover adequada formação do sistema radicial e proteger as raízes de danos mecânicos e desidratação, além de contribuir para a máxima sobrevivência e crescimento no campo. Nos últimos anos, novos recipientes têm sido desenvolvidos a fim de contribuir para a produção de mudas florestais com melhor padrão de qualidade. Estudos comparativos entre os diversos recipientes têm sido objeto de trabalhos experimentais, desenvolvidos por muitos pesquisadores (FARIAS JÚNIOR et al., 2007; PEREIRA et al., 2010; COÊLHO et al., 2013).

Todavia, esses estudos limitam-se à avaliação de mudas produzidas em recipientes apenas em viveiro, portanto, carecendo de informações a respeito do efeito dos mesmos no estabelecimento e ritmo de crescimento das plantas após o plantio, em particular com a espécie em estudo.

Nesse sentido, a presente pesquisa teve como objetivo avaliar a qualidade morfofisiológica de mudas de nim indiano em diferentes recipientes, visando alcançar maiore produtividade no campo. 


\section{MATERIAL E MÉTODOS}

\section{Localização dos experimentos}

O presente estudo foi conduzido em três etapas. A primeira constou da produção das mudas no Viveiro de Pesquisa da Universidade Estadual do Sudoeste da Bahia (UESB), campus de Vitória da Conquista, e avaliação das características morfológicas no Laboratório de Silvicultura e Taxonomia. A segunda etapa constou da avaliação do potencial de regeneração de raízes (PRR), desenvolvida em casa de vegetação na UESB. A terceira etapa foi desenvolvida no Campo Agropecuário da UESB, que se encontra nas coordenadas geográficas de $14^{\circ} 51^{\prime}$ ' de latitude sul e $40^{\circ} 50^{\prime}$ de longitude oeste. A região apresenta relevo plano a levemente ondulado e precipitação pluviométrica variando de 700 a $1.100 \mathrm{~mm}$ anuais, sendo os meses mais chuvosos de novembro a março.

A temperatura média anual é de $21{ }^{\circ} \mathrm{C}$. O solo da área experimental é classificado como Latossolo Vermelho Amarelo distrófico, com textura média e de topografia plana e boa drenagem. A vegetação característica e predominante da região é a Floresta Estacional Semidecidual Montana (NOVAES et al., 2008).

\section{Recipientes utilizados na produção das mudas \\ Sacolas plásticas}

Foram utilizadas sacolas plásticas com dois tamanhos. O primeiro com dimensões de $22 \mathrm{~cm}$ de altura e $11 \mathrm{~cm}$ de diâmetro, com capacidade volumétrica para $382 \mathrm{~cm}^{3}$. O segundo tamanho teve as dimensões de $15 \mathrm{~cm}$ de altura e $7,0 \mathrm{~cm}$ de largura, com $165 \mathrm{~cm}^{3}$ de capacidade volumétrica de substrato.

Tubete

Foram utilizados dois modelos de tubetes. O primeiro modelo apresenta dimensões de $19 \mathrm{~cm}$ de altura e $5,0 \mathrm{~cm}$ de diâmetro na parte superior, apresentando o fundo aberto com aproximadamente $1,0 \mathrm{~cm}$ e com $288 \mathrm{~cm}^{3}$ de capacidade volumétrica. O segundo modelo teve as dimensões de $12,5 \mathrm{~cm}$ de altura, $3,0 \mathrm{~cm}$ de diâmetro na parte superior e 1,0 cm de diâmetro na parte inferior, com capacidade volumétrica para $55 \mathrm{~cm}^{3}$.

\section{Substrato utilizado}

Para enchimento das sacolas plásticas, foi utilizado o substrato composto de $40 \%$ de matéria orgânica, 30\% de terra de subsolo e 30\% de areia, acrescidos do adubo 04-30-16 + Zn (200 g/litro). Para enchimento dos tubetes, foi usado o substrato da marca comercial Mecplant, constituído de casca de pinus bioestabilizada, acrescido do adubo de liberação lenta osmocot (5,0 g/litro). As sementes usadas no presente trabalho foram adquiridas junto à empresa Nimbahia. Antes da semeadura direta nos recipientes, foi efetuada uma pré-germinação, visando maximizar o processo de germinação, utilizando-se um saco de estopa dobrado, no qual as sementes foram distribuídas em uma única camada e regadas durante 12 dias. Ao final desse período, quando as sementes apresentaram radículas de aproximadamente cinco milímetros, elas foram definitivamente semeadas nos respectivos recipientes. Após a semeadura, as mudas foram irrigadas duas vezes ao dia (manhã e tarde), mediante sistema de irrigação por aspersores.

\section{Avaliação morfológica}

Aos quatro meses de idade, as mudas foram retiradas de forma aleatória do viveiro e suas características morfológicas avaliadas: a) altura da parte aérea $(\mathrm{H})$; b) diâmetro de colo (D); c) relação $\mathrm{H} / \mathrm{D}$; d) matéria fresca da parte aérea; e) matéria fresca do sistema radicial; f) matéria fresca total; g) matéria seca da parte aérea; h) matéria seca do sistema radicial; e i) matéria seca total. Para essa avaliação, foi efetuada uma lavagem do sistema radicial das mudas, eliminando todos os resíduos de substrato aderidos às raízes. Após esse procedimento, as mudas foram postas sobre folhas de jornal na bancada do laboratório, por um período de 12 horas, para perda do excesso de água. Após esse tempo, foram efetuadas as medições da altura da parte aérea e do diâmetro de colo, utilizando-se régua graduada e paquímetro digital. Em seguida, procedeu-se à divisão entre a haste e o sistema radicial, visando a determinação de pesos de matéria fresca e seca da parte aérea, de raízes e total. Foi efetuada a separação dessas duas partes muda por muda, sendo acondicionadas em embalagens e levadas à secagem em estufa 
previamente aquecida a $75{ }^{\circ} \mathrm{C}$, conforme orientações de Schuurman e Goedewaagen (1971), citados por Böhm (1979). Na estufa, as embalagens foram abertas para facilitar a perda de umidade, e o material permaneceu por um período de 24 horas, atingindo peso constante. As pesagens foram efetuadas após esfriamento, utilizando-se balança digital.

\section{Avaliação do potencial de regeneração das raízes (PRR)}

Para essa avaliação, depois de retiradas do viveiro após quatro meses da semeadura, as mudas foram submetidas a lavagem cuidadosa do sistema radicial e, posteriormente, submetidas a poda das raízes laterais a uma distância de quatro centímetros do eixo da raiz pivotante, a qual também foi podada a uma distância de $12 \mathrm{~cm}$ do colo. Utilizou-se como recipientes tubos plásticos transparentes com dimensões de $25 \mathrm{~cm}$ de altura por $10 \mathrm{~cm}$ de largura e cobertos com lona preta. O PRR foi avaliado 30 dias após o transplantio das mudas para os tubos por meio da determinação do número total de raízes regeneradas e visíveis nas paredes desses recipientes. O procedimento adotado constou da marcação, por meio de um pincel atômico, de pontos nas paredes transparentes dos recipientes, exatamente nos locais tocados pelas extremidades das raízes novas regeneradas.

\section{Avaliação do desempenho das mudas no campo}

Para a instalação do experimento de campo o preparo do solo consistiu de gradagem e abertura de sulcos nas dimensões de $35,0 \mathrm{~cm}$ x $35,0 \mathrm{~cm}$. O plantio foi realizado em abril de 2010 , sendo efetuado manualmente na linha do sulco, obedecendo o espaçamento de 3,0 x 3,0 m, sendo efetuada em cada ponto de plantio uma adubação com $100 \mathrm{~g}$ de superfosfato simples. Em seguida, as mudas foram irrigadas com a adição de dois a três litros de água por planta. Periodicamente, foi realizada a eliminação do matocompetição, através de roçada, e o combate a formigas-cortadeiras, utilizando isca formicida. Para evitar o efeito de borda, foi efetuado o plantio de duas fileiras com plantas da mesma espécie circundando toda a área experimental.

A avaliação do desempenho das mudas no campo constou da mensuração da altura da parte aérea e do diâmetro ao nível do solo, em intervalos de três meses, durante 15 meses, utilizando-se vara graduada e paquímetro digital, respectivamente. O índice de sobrevivência foi avaliado 90 dias após o plantio. Foi avaliado ainda o ritmo de crescimento das mudas no campo, por meio de análise de regressão, considerando a altura da parte aérea e o diâmetro de colo, utilizando-se o programa científico de ajuste de curvas SigmaPlot ${ }^{\circledR}$ v. 12.0 .

\section{Tratamentos e procedimentos estatísticos}

Foram considerados quatro tratamentos, envolvendo dois sistemas de produção de mudas (sacolas plásticas e tubetes): T1 - sacola plástica $\left(382 \mathrm{~cm}^{3}\right) ; \mathrm{T} 2$ - sacola plástica $\left(165 \mathrm{~cm}^{3}\right)$; T3 - tubete $\left(288 \mathrm{~cm}^{3}\right)$; T4 - tubete $\left(55 \mathrm{~cm}^{3}\right)$. A etapa referente à produção das mudas no viveiro foi instalada obedecendo-se a um delineamento experimental inteiramente ao acaso, com quatro tratamentos e cinco repetições, perfazendo um total de 20 parcelas. Cada parcela constou de 100 mudas, totalizando 2000 mudas no experimento. Para avaliação do PRR, foram utilizadas 40 mudas, distribuídas nos quatro tratamentos, compondo assim dez repetições por tratamento com parcelas constituídas de uma muda por tubo. No campo, utilizou-se o delineamento experimental em blocos casualizados, com quatro tratamentos e cinco repetições, totalizando 20 parcelas, as quais constaram de seis mudas cada, perfazendo um total de 120 mudas no experimento. Todos os resultados obtidos foram submetidos a análise de variância e as médias foram comparadas pelo teste de Duncan a 5\% de probabilidade.

\section{RESULTADOS E DISCUSSÃO}

\section{Altura da parte aérea (H), diâmetro de colo (D) e relação H/D}

Analisando os dados da tabela 1, observou-se que a sacola plástica com $382 \mathrm{~cm}^{3}$ de capacidade volumétrica de substrato apresentou as maiores médias para todas as características, diferindo pelo teste de Duncan dos demais tratamentos. Esse resultado pode estar associado ao maior volume de substrato no recipiente e, consequentemente, à maior disponibilidade de nutrientes (BARROSO et al., 2000). Bomfim et al. (2009), ao estudarem a qualidade morfológica de mudas de madeira nova (Pterogyne nitens Tull.) produzidas 
em tubetes e sacolas plásticas, obtiveram resultados semelhantes para essas características. Não houve diferença estatística entre os dois sistemas de tubete estudados, porém as menores médias para as características avaliadas foram verificadas em mudas produzidas em tubetes de $55 \mathrm{~cm}^{3}$, o que pode ser atribuído à sua menor dimensão, promovendo maior restrição radicial. Quanto à avaliação da relação $\mathrm{H} / \mathrm{D}$, característica que determina o equilíbrio de desenvolvimento das mudas, constatou-se que a maior relação coube ao tratamento correspondente à sacola plástica $382 \mathrm{~cm}^{3}$, diferindo estatisticamente dos demais tratamentos. Os menores valores dessa relação foram constatados em mudas produzidas no sistema de tubetes.

Tabela 1. Valores médios de altura da parte aérea (H), diâmetro de colo (D) e relação H/D de mudas de nim indiano (Azadirachta indica), quatro meses após a semeadura.

Table 1. Medium values of the stem height $(\mathrm{H})$, root-collar diameter (D) and H/D ratio of Indian Lilac (Azadirachta indica) seedlings, four months after sowing.

\begin{tabular}{lccc}
\hline Recipientes & Altura $(\mathbf{c m})$ & Diâmetro $(\mathbf{m m})$ & H/D \\
\hline Sacola plástica $382 \mathrm{~cm}^{3}$ & $35,62 \mathrm{a}$ & $4,17 \mathrm{a}$ & $8,56 \mathrm{a}$ \\
Sacola plástica $165 \mathrm{~cm}^{3}$ & $22,12 \mathrm{~b}$ & $3,25 \mathrm{~b}$ & $6,78 \mathrm{~b}$ \\
Tubete $288 \mathrm{~cm}^{3}$ & $11,24 \mathrm{c}$ & $2,65 \mathrm{c}$ & $4,33 \mathrm{c}$ \\
Tubete $55 \mathrm{~cm}^{3}$ & $9,28 \mathrm{c}$ & $2,49 \mathrm{c}$ & $3,81 \mathrm{c}$ \\
\hline CV \% & 8,82 & 5,11 & 8,16 \\
\hline
\end{tabular}

Médias seguidas de mesma letra na coluna não diferem entre si pelo teste de Duncan a 5\% de probabilidade.

\section{Avaliação de pesos de matéria fresca e seca}

Os dados da tabela 2 mostram que as mudas produzidas em sacolas plásticas $382 \mathrm{~cm}^{3}$ apresentaram as maiores médias de pesos de matéria fresca para todas as características pesquisadas, diferindo estatisticamente dos demais tratamentos. As menores médias couberam ao sistema de produção de mudas em tubetes $55 \mathrm{~cm}^{3}$, evidenciando limitações ao crescimento das raízes e consequentemente das mudas nesse recipiente. Contudo, em relação ao peso de matéria fresca da parte aérea e total, os tratamentos referentes aos tubetes $288 \mathrm{~cm}^{3}$ e $55 \mathrm{~cm}^{3}$ não apresentaram diferença estatística. Dados similares foram encontrados por Samôr (1999), estudando o comportamento de mudas de Sesbania virgata (Cav.) e Anadenanthera macrocarpa (Benth.) Brenan em diferentes recipientes e substratos.

Tabela 2. Valores médios de peso da matéria fresca das partes aérea, radicial e total de mudas de nim indiano (Azadirachta indica), quatro meses após a semeadura.

Table 2. Medium values of weight of the fresh matter of the stem, root and total of the seedlings of Indian Lilac (Azadirachta indica), four months after sowing.

\begin{tabular}{lccr}
\hline \multirow{2}{*}{ Recipientes } & \multicolumn{3}{c}{ Peso de matéria fresca $(\mathbf{g})$} \\
\cline { 2 - 4 } & Parte aérea & Raiz & Total \\
\hline Sacola plástica $382 \mathrm{~cm}^{3}$ & $8,23 \mathrm{a}$ & $1,88 \mathrm{a}$ & $10,12 \mathrm{a}$ \\
Sacola plástica $165 \mathrm{~cm}^{3}$ & $3,05 \mathrm{~b}$ & $0,98 \mathrm{~b}$ & $4,04 \mathrm{~b}$ \\
Tubete $288 \mathrm{~cm}^{3}$ & $0,99 \mathrm{c}$ & $0,57 \mathrm{c}$ & $1,57 \mathrm{c}$ \\
Tubete $55 \mathrm{~cm}^{3}$ & $0,54 \mathrm{c}$ & $0,33 \mathrm{~d}$ & $0,88 \mathrm{c}$ \\
\hline CV \% & 13,14 & 17,96 & 12,89 \\
\hline
\end{tabular}

Médias seguidas de mesma letra na coluna não diferem entre si pelo teste de Duncan a 5\% de probabilidade.

Quanto aos dados constantes da tabela 3, as maiores médias foram verificadas em mudas produzidas em sacolas plásticas $382 \mathrm{~cm}^{3}$, diferindo estatisticamente dos demais tratamentos, o que indica uma muda com maior vigor e propensa a suportar condições adversas no campo após o plantio. Para o peso de matéria seca da parte aérea, não foi constatada diferença significativa entre os tratamentos referentes aos tubetes. Segundo Carneiro (1995), o peso de matéria seca da parte aérea é uma boa indicação da capacidade de resistência das mudas no campo. Ainda conforme esse autor, em relação ao peso de raízes, tanto o peso de matéria fresca quanto o de matéria seca correspondem sempre a valores muito pequenos, mesmo que as mudas apresentem grande volume de raízes finas, com alta quantidade de pelos absorventes. Contudo, sob o ponto de vista fisiológico, esse volume de raízes é de fundamental importância na sobrevivência e crescimento inicial das mudas, dada a sua função no processo de absorção de água e nutrientes do solo após o plantio. 
Tabela 3. Valores médios de peso da matéria seca das partes aérea, radicial e total de mudas de nim indiano (Azadirachta indica), quatro meses após a semeadura.

Table 3. Medium values of weight of the dry matter of the stem, root and total of the seedlings of Indian Lilac (Azadirachta indica), four months after sowing.

\begin{tabular}{lccc}
\hline \multirow{2}{*}{ Recipientes } & \multicolumn{3}{c}{ Peso de matéria seca $(\mathbf{g})$} \\
\cline { 2 - 4 } & Parte aérea & Raiz & Total \\
\hline Sacola plástica $382 \mathrm{~cm}^{3}$ & $3,83 \mathrm{a}$ & $1,01 \mathrm{a}$ & $4,85 \mathrm{a}$ \\
Sacola plástica $165 \mathrm{~cm}^{3}$ & $1,53 \mathrm{~b}$ & $0,61 \mathrm{~b}$ & $2,15 \mathrm{~b}$ \\
Tubete $288 \mathrm{~cm}^{3}$ & $0,61 \mathrm{c}$ & $0,42 \mathrm{c}$ & $1,04 \mathrm{c}$ \\
Tubete $55 \mathrm{~cm}^{3}$ & $0,37 \mathrm{c}$ & $0,24 \mathrm{~d}$ & $0,62 \mathrm{~d}$ \\
\hline CV\% & 13,22 & 17,16 & 13,16 \\
\hline
\end{tabular}

Médias seguidas de mesma letra na coluna não diferem entre si pelo teste de Duncan a 5\% de probabilidade.

\section{Avaliação do potencial de regeneração de raízes}

Pode-se observar, conforme a tabela 4 , que as mudas de nim indiano produzidas em tubetes $288 \mathrm{~cm}^{3}$ apresentaram um maior número de raízes regeneradas, apesar de não haver diferença estatística em relação aos tratamentos correspondentes a sacolas plásticas $382 \mathrm{~cm}^{3}$ e tubetes $55 \mathrm{~cm}^{3}$. Já as mudas produzidas no sistema de sacolas plásticas de $165 \mathrm{~cm}^{3}$ obtiveram médias inferiores em relação aos demais recipientes, porém, estatisticamente não se observou diferença entre este tratamento e os referentes à sacola plástica de maior dimensão e ao tubete de menor capacidade volumétrica. Ao associar os resultados do PRR com a avaliação do campo, pode-se observar que as mudas que apresentaram maior capacidade de regeneração de suas raízes também apresentaram maior sobrevivência no campo, demonstrando a importância do teste na avaliação do PRR. Segundo Carneiro (1995), o potencial de regeneração de raízes é uma característica que prognostica o percentual de sobrevivência e o crescimento após o plantio.

Tabela 4. Valores médios do potencial de regeneração de raízes (PRR) de mudas de nim indiano (Azadirachta indica).

Table 4. Medium values of root regeneration potential (RRP) of the seedlings of Indian Lilac (Azadirachta indica).

\begin{tabular}{lc}
\hline Recipientes & Número de Raízes \\
\hline Sacola plástica $382 \mathrm{~cm}^{3}$ & $126,3 \mathrm{ab}$ \\
Sacola plástica $165 \mathrm{~cm}^{3}$ & $89,0 \mathrm{~b}$ \\
Tubete $288 \mathrm{~cm}^{3}$ & $204,1 \mathrm{a}$ \\
Tubete $55 \mathrm{~cm}^{3}$ & $161,5 \mathrm{ab}$ \\
\hline CV\% & 31,15 \\
\hline
\end{tabular}

Médias seguidas de mesma letra na coluna não diferem entre si pelo teste de Duncan a 5\% de probabilidade.

\section{Avaliação do desempenho das mudas no campo}

Índice de sobrevivência

Conforme dados constantes da tabela 5 , verificou-se $100 \%$ de sobrevivência para a maioria dos tratamentos avaliados 90 dias após o plantio. O menor índice de sobrevivência coube ao tratamento sacola plástica $165 \mathrm{~cm}^{3}$, o qual produziu mudas com menor potencial de regeneração de raízes.

Tabela 5. Valores médios do índice de sobrevivência de mudas de nim indiano (Azadirachta indica), 90 dias após o plantio.

Table 5. Medium values of the survival index of seedlings of Indian Lilac (Azadirachta indica), 90 days after planting.

\begin{tabular}{lc}
\hline Recipientes & Sobrevivência (\%) \\
\hline Sacola plástica $382 \mathrm{~cm}^{3}$ & 100 \\
Sacola plástica $165 \mathrm{~cm}^{3}$ & 90 \\
Tubete $288 \mathrm{~cm}^{3}$ & 100 \\
Tubete $55 \mathrm{~cm}^{3}$ & 100 \\
\hline
\end{tabular}

\section{Desempenho em altura e diâmetro}

Conforme a tabela 6, o teste de Duncan constatou, aos 15 meses após o plantio, que as mudas produzidas em sacolas plásticas com capacidade volumétrica de $382 \mathrm{~cm}^{3}$ apresentaram as maiores médias 
de altura da parte aérea, não se verificando diferença significativa em relação ao sistema de tubetes $288 \mathrm{~cm}^{3}$, o qual apresentou, no viveiro, o maior potencial de regeneração de raízes, evidenciando, assim, a eficiência dessa característica na avaliação da qualidade de mudas florestais. Já as menores médias referentes a essa característica foram encontradas no sistema de produção de mudas em tubetes $55 \mathrm{~cm}^{3}$, o qual não apresentou diferença estatística em relação ao sistema de produção em sacolas plásticas de $165 \mathrm{~cm}^{3}$ e tubetes $288 \mathrm{~cm}^{3}$. Resultados similares foram encontrados por Malavasi e Malavasi (2006), os quais, trabalhando com mudas de Cordia trichotoma (Vell.) Arrab. ex Steud e Jacaranda micranta Cham., constataram menores médias de altura quando produzidas em tubetes de $55 \mathrm{~cm}^{3}$. Por outro lado, Barros et al. (1978), ao estudarem os efeitos de recipientes na sobrevivência e crescimento de mudas de Eucalyptus grandis Hill ex Maiden, reportaram, após 108 dias do plantio, que as maiores médias em altura foram obtidas de mudas produzidas em sacolas plásticas $(6,0 \times 10 \mathrm{~cm})$.

Quanto ao diâmetro ao nível do solo, mudas produzidas em sacolas plásticas de $382 \mathrm{~cm}^{3}$ apresentaram as maiores médias, sendo constatada, pelo teste de Duncan, diferença significativa em relação aos demais tratamentos. De acordo com Barnett (1983), a altura e o diâmetro de colo são os indicadores da qualidade de mudas florestais mais confiáveis quando o objetivo é a formação de povoamentos florestais produtivos. As menores médias para essa característica foram obtidas em mudas produzidas em tubetes de $55 \mathrm{~cm}^{3}$. Trabalhando com características indicadoras da qualidade de mudas de Jatropha curcas L., Ajala et al. (2012) encontraram resultados similares.

Tabela 6. Valores médios de altura da parte aérea e diâmetro ao nível do solo de plantas de nim indiano (Azadirachta indica), 15 meses após o plantio.

Table 6. Medium values of height of the stem and diameter at the soil level of plants of Indian Lilac (Azadirachta indica), 15 months after planting.

\begin{tabular}{lcc}
\hline Recipientes & Altura $(\mathbf{c m})$ & Diâmetro $(\mathbf{m m})$ \\
\hline Sacola plástica $382 \mathrm{~cm}^{3}$ & $178,10 \mathrm{a}$ & $43,99 \mathrm{a}$ \\
Sacola plástica $165 \mathrm{~cm}^{3}$ & $144,74 \mathrm{~b}$ & $33,46 \mathrm{~b}$ \\
Tubete $288 \mathrm{~cm}^{3}$ & $153,30 \mathrm{ab}$ & $33,24 \mathrm{~b}$ \\
Tubete $55 \mathrm{~cm}^{3}$ & $126,41 \mathrm{~b}$ & $29,10 \mathrm{~b}$ \\
\hline CV\% & 14,46 & 17,30 \\
\hline
\end{tabular}

Médias seguidas de mesma letra na coluna não diferem entre si pelo teste de Duncan a 5\% de probabilidade.

Para todos os tratamentos, o modelo matemático que melhor explicou no campo o ritmo de crescimento das plantas em altura e diâmetro ao nível do solo, em função da idade, foi o exponencial, representado pela equação: $\mathrm{Y}=\mathrm{ab}{ }^{\text {Idade }}$.

Os resultados, obtidos aos 15 meses após o plantio, demonstraram um maior ritmo de crescimento em altura (Figura 1) de mudas produzidas em sacolas plásticas de $382 \mathrm{~cm}^{3}$, cuja equação de regressão ajustada para esse tratamento e com $\mathrm{R}^{2}=0,96$ encontra-se a seguir: $\mathrm{Y}=45,1959 * 1,1010^{\text {Idade }}$. Todavia, nota-se que este pode ser superado em um período maior, por mudas produzidas em tubetes de $288 \mathrm{~cm}^{3}$, que apresentaram um ritmo de crescimento com tendência de maior incremento em altura. Os resultados do PRR corroboram essa hipótese. Leles et al. (2000), ao estudarem a qualidade de mudas de Eucalyptus camaldulensis, E. grandis e E. pellita encontraram resultados similares. O menor ritmo de crescimento em altura foi verificado para plantas produzidas no sistema tubete $55 \mathrm{~cm}^{3}$.

Observando-se a figura 2, pode-se inferir que o desenvolvimento inicial das mudas oriundas do sistema de sacolas plásticas $382 \mathrm{~cm}^{3}$ destacou-se dos demais, apresentando um ritmo de crescimento em diâmetro bastante acentuado. Pode-se observar, ainda, um maior ritmo de crescimento de mudas produzidas em tubetes de $288 \mathrm{~cm}^{3}$, com tendência de acompanhar, e até mesmo superar, em um período maior, as mudas produzidas em sacolas plásticas $165 \mathrm{~cm}^{3}$. Resultados similares foram obtidos por Bomfim et al. (2009), ao trabalharem com mudas de madeira nova. O menor ritmo de crescimento em diâmetro também foi verificado em mudas produzidas em tubete com capacidade volumétrica de $55 \mathrm{~cm}^{3}$, o que pode ser atribuído, conforme Novaes et al. (2002), ao fato de esse recipiente ter provocado, no viveiro, restrições à raízes das mudas, o que persistiu após o plantio no campo. 


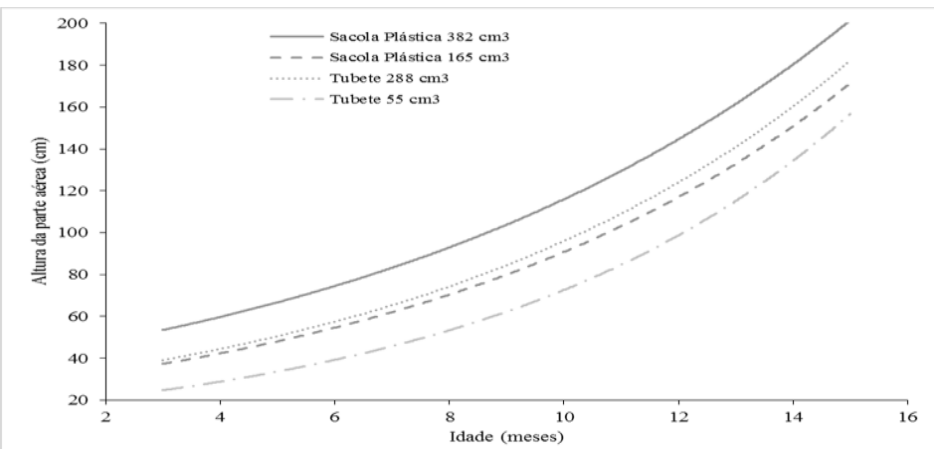

Figura 1. Crescimento em altura de mudas de nim indiano (Azadirachta indica) aos 15 meses após o plantio.

Figure 1. Height growth of seedlings of Indian Lilac (Azadirachta indica) at 15 months after planting.



Figura 2. Crescimento em diâmetro de mudas de nim indiano (Azadirachta indica) aos 15 meses após o plantio.

Figure 2. Diameter growth of seedlings of Indian Lilac (Azadirachta indica) at 15 months after planting.

\section{CONCLUSÕES}

- O sistema de produção de mudas em sacolas plásticas com $382 \mathrm{~cm}^{3}$, para todas as características morfológicas avaliadas e desempenho no campo, 15 meses após o plantio, foi superior aos demais métodos utilizados nesta pesquisa, demonstrando, assim, a sua eficiência na produção de mudas de nim indiano.

- O potencial de regeneração de raízes (PRR), avaliado em tubos, demonstrou ser uma característica confiável na determinação da qualidade de mudas produzidas em tubetes com $288 \mathrm{~cm}^{3}$.

\section{REFERÊNCIAS}

AJALA, M. C.; AQUINO, N. F.; MALAVASI, U. C.; MALAVASI, M. de M. Efeito do volume do recipiente na produção de mudas e crescimento inicial de Jatropha curcas L. no oeste paranaense. Semina: Ciências Agrárias, Londrina, PR, v. 33, n. 6, p. 2039 - 2046, 2012.

BARNETT, J. P. Relating seedling morphology of container-grown southern pines to field success. Separata de: CONVENTION OF THE SOCIETY OF AMERICAN FORESTERS (1983: Portland). Proceedings... New Orleans: USDA. For. Serv. Southern Forest Experiments Station, 1983. p. 405 - 407. 
BARROS, N. F.; BRANDI, R. M.; COUTO, L.; REZENDE, G. C. Efeitos de recipientes na sobrevivência e crescimento de mudas de Eucaliptus grandis W. Hill ex Maiden, no viveiro e no campo. Árvore, Viçosa, MG, v. 2, n. 2, p. 141 - 151, 1978.

BARROSO, D. G.; CARNEIRO, J. G. A.; LELES, P. S. S. Qualidade de mudas de Eucalyptus camaldulensis e E. urophylla produzidas em tubetes e em blocos prensados, com diferentes substratos. Floresta e Ambiente, Seropédica, RJ, v. 7, n. 1, p. 238 - 250, 2000.

BITTENCOURT, A. M.; SANTOS, A. J.; HOEFLICH, V. A.; BERGER, R. O cultivo do nim indiano (Azadirachta indica A. Juss.): uma visão econômica. Floresta, Curitiba, PR, v. 39, n. 3, p. 629 - 642, 2009.

BÖHM, W. Methods of studying root systems. Berlin: Springer-Verlag, 1979. 188 p.

BOMFIM, A. A.; NOVAES, A. B.; SÃO JOSÉ, A. R.; GRISI, F. A. Avaliação morfológica de mudas de madeira-nova (Pterogyne nitens Tull.) produzidas em tubetes e sacos plásticos e de seu desempenho no campo. Floresta, Curitiba, PR, v. 39, n. 1, p. 33 - 40, 2009.

CARNEIRO, J. G. A. Produção e controle de qualidade de mudas florestais. Curitiba: Campos/UENF. UFPR/FUPEF, 1995. $451 \mathrm{p}$.

COÊLHO, I. A. M.; BOTELHO, A. V. F.; LOPES, I. S.; COELHO, O. A. M.; SERPA, P. R. K.; PASSOS, M. A. A. Efeito de recipientes e tipo de substratos na qualidade das mudas de Poincianella pyramidales (Tull.) L. P. Queiroz. Scientia Plena, Sergipe, v. 9, n. 5, 5 p., 2013.

FARIAS JÚNIOR, J. A.; CUNHA, M. C. L.; FARIAS, S. G. G.; MENEZES JÚNIOR, J. C. Crescimento inicial de mudas de turco sob diferentes tipos de recipientes e níveis de luminosidade. Revista Brasileira de Ciências Agrárias, Recife, PE, v. 2, n. 3, p. 228 - 232, 2007.

GOOR, A. Y. Métodos de plantación forestal em zonas áridas. FAO: Cuadernos de Fomento Forestal, 16. Roma, 1964. 265 p.

LELES, P. S. S.; CARNEIRO, J. G. A.; BARROSO, D. G.; MORGADO, I. F. Qualidade de mudas de Eucalyptus spp. produzidas em blocos prensados e em tubetes. Árvore, Viçosa, MG, v. 24, n. 1, p. 13 20, 2000.

LORENZI, H.; SOUZA, H. M. de; TORRES, M. A. V.; BACHER, L. B. Árvores exóticas no Brasil: madeireiras, ornamentais e aromáticas. Nova Odessa, SP: Instituto Plantarum, 2003. 384 p.

MALAVASI, U. C.; MALAVASI, M. M. Efeito do volume do tubete no crescimento inicial de plântulas de Cordia trichotoma (Vell.) Arrab. ex Steud e Jacaranda micranta Cham. Ciência Florestal, Santa Maria, v. 16, n. 1, p. 11 - 16, 2006.

MARTINEZ, S. S.; RAMOS, A. L. M.; CARNEIRO, S. M. T. P. G.; BRECHELT, A. O nim, Azadirachta indica: natureza, usos múltiplos, produção. Londrina: IAPAR, 2002. 142 p.

MOURÃO, S. A.; ZANUNCIO, J. C.; SILVA, J. C. T; JHAM, G. N. Nim indiano (Azadirachta indica): mil utilidades. Boletim de Extensão, 47. Viçosa: UFV, 2004. 26 p., il. Bibliografia: ISSN 1415-692X.

NEVES, B. P.; OLIVEIRA, I. P.; NOGUEIRA, J. C. M. Cultivo e utilização do nim indiano. Circular Técnica, nº 62 - EMBRAPA. Santo Antônio de Goiás, GO, dezembro, 2003.

NOVAES, A. B.; LONGUINHOS, M. A. A.; RODRIGUES, J.; SANTOS, I. F. dos; GUSMÃO, J. C. Caracterização e demanda florestal da região sudoeste da Bahia. In: SANTOS, A. F. dos; NOVAES, A. B. de; SANTOS, I. F. dos; LONGUinhos, M. A. A. (Org.). Memórias do II Simpósio sobre Reflorestamento na Região Sudoeste da Bahia. 1. ed. Colombo: Embrapa Florestas, v. 1, 2008, p. 25 43.

NOVAES, A. B.; CARNEIRO, J. G. A.; BARROSO, D. G.; LELES, P. S. S. Avaliação do potencial de regeneração de raízes de mudas de Pinus taeda L., produzidas em diferentes tipos de recipientes, e o seu desempenho no campo. Árvore, Viçosa, MG, v. 26, n. 6, p. 675 - 681, 2002. 
OLIVEIRA, I. P.; NEVES, B. P.; MOREIRA, F. P.; COSTA, K. A. P. Manejo sustentável e nutrição mineral do nim indiano. Comunicado Técnico no 110 - EMBRAPA. Santo Antônio de Goiás, GO, dezembro, 2005.

PEREIRA, P. C.; MElO, B.; FREITAS, R. S.; TOMAZ, M. A.; TEIXEIRA, I. R. Tamanho de recipientes e tipos de substrato na qualidade de mudas de tamarindeiro. Revista Verde, Mossoró, RN, v. 5, n. 3, p. 136 - 142, 2010.

SAMÔR, O. J. M. Comportamento de mudas de Sesbania virgata e Anadenanthera macrocarpa produzidas em diferentes recipientes e substratos, destinadas à recuperação de áreas degradadas pela extração de argila. 78 f. Dissertação (Mestrado em Produção Vegetal) - Universidade Estadual do Norte Fluminense, Campos dos Goytacazes, 1999.

SOARES, F. P.; PAIVA, R.; NOGUEIRA, R. C.; OLIVEIRA, L. M.; PAIVA, P. D. O.; SILVA, D. R. G. Cultivo e usos do nim (Azadirachta indica A. Juss). Boletim Agropecuário, UFLA, n. 68, p. 1 - 14. s.d.

VITORINO, A. C. T.; ROSA JÚNIOR, E. J.; DANIEL, O. Influência de diferentes combinações de doses de n-p-k no crescimento de mudas de Eucalyptus grandis produzidas em tubetes. Revista Científica. UFMS, Campo Grande, v. 3, n. 1, p. 07 - 13, 1996. 